\title{
A Study on Paintings Art Style Using Multi-Cues
}

\author{
Fang Lu \\ Academy of Fine Arts, Dalian University, Dalian, China, 116622 \\ E-mail:shining_112@live.cn
}

Keywords: Image classification; Painting; Art style; Blank leaving.

\begin{abstract}
Visual characteristics of paintings display high-level semantic concept: art style to the viewers. Classification of art style depends mainly on human knowledge and experience, which remains a big challenge for computer vision. In this paper, based on careful studies on art literature, we propose a simple but effective method to automatically identify the art style between the Chinese wash painting and the foreign art painting. The efficiency of our method lies on that three cues: color contrast, blank-leaving and uniformity of illumination are utilized to recognize the art style of one image. Experiments results show that, our method outperforms stateof- the-art approaches, yielding higher precision while requiring less computation time. Using the cues presented in this paper, our method can successfully identify whether one painting belongs to Chinese or foreign art style.
\end{abstract}

\section{Introduction}

The on-going research of computer vision algorithms mainly focus on analyzing the content of the image [1]. Recent-developed classification methods [2] often focus on the low-level content of the image, for example, shapes, key points, etc. Unfortunately, the art style is high-level semantic concept and is hard to be represented only by shapes or feature points. Nowadays, one of the more challenging tasks for computers is the analysis and representation of the art style of the images or paintings. The motivation for recognizing the art style of a painting is not only to build the bridge between computer vision and human perception but also to build a bridge between computer vision and art works. Also, to see if a computer can perform what has traditionally been a human-only task is an interesting problem in itself.

Based on art philosophy, this paper proposes a simple but effective method to classify the different art style between Chinese wash painting and foreign art painting. Throughout numerous studies on art literature, we have found that in Chinese wash painting [4] [5], the main art components are brushwork, color and blank-leaving, as opposed to brushwork, color and illumination in foreign art painting[6] [7]. Based on this observation, we propose an algorithm using the color contrast, blank-leaving and the uniformity of illumination as the features to classify art styles, and then the SVM is used as the classifier. The experiments prove that, in term of classification accuracy and runtime, our method is superior to state-of-the-art image classification method. Our work is not meant to provide a full solution, but rather it aims to inspire more interests in this new and fundamentally important and challenging research direction.

\section{Color Contrast}

The color of Chinese wash painting stresses generalityand decorative. In the Chinese wash paintings, the colorrelationship of object content is described by the shade of inkchanges. However, the 
foreign artist pay more attention onthe exactly reappearance of what they see, and the colorchanges abundantly. Intuition, the color contrast will be oneof the characters between the two different art styles. In thispaper, we first compute the color difference of one pixel inimage I as:

$$
d\left(I_{i}\right)=\sum_{j \in N(i)}\left|I_{i}-I_{j}\right|
$$

Where $\left|I_{i}-I_{j}\right|$ represents the absolute difference betweenone pixel $i$ and its neighboring pixel $j$ and $\mathrm{N}(\mathrm{j})$ denotes theneighborhood around pixel $\mathrm{j}$.

Then, we map this color difference into a histogram,suppose the maximum of the histogram value is $\mathrm{m}$.So thefinal color contrast value is the $\log$ of $\mathrm{m}$

$$
f_{1}=\log m
$$

We combine all color contrast value of the $\mathrm{M}$ training images to form the histogram. Figure 1 shows the color contrast histogram using above method. Obviously, using the mean value of this histogram, we can successfully classify the art style between Chinese wash painting and foreign art painting, since we have found from our experiment that a mean value 11.36 for the Chinese wash painting and 10.09 for the foreign art paintings.It is obviously that, in most cases, the $\mathrm{f}_{1}$ value of Chinese wash painting is larger than that of foreign art painting.

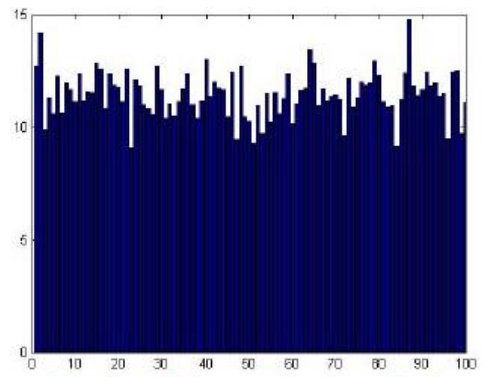

(a) the Chinese wash paintings

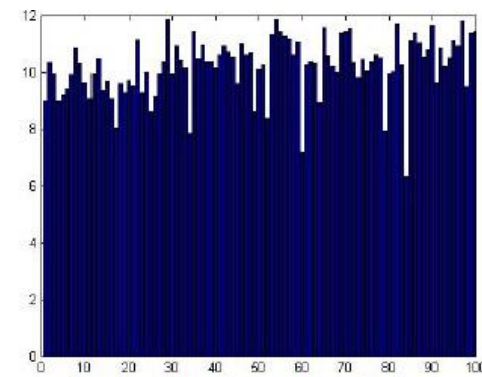

(b) the foreign art paintings

Figure.1The color difference histogram

\section{Feature Extration}

We have constructed a database consisting of 200 images, each art style images is 100. To objectively and correctly measure our algorithm, the database is subtly designed as follows: for the Chinese wash paintings, the content includes person, scene, flower, animal, mountain, bamboo and so on ; for the foreign art paintings, images differ according to region style: Baroque and Rococo, classicism and so on, and the content includes religious paintings, landscapes, portrait and so on. Figure 1 shows several images with each art style from the dataset. From the sample images, we could see the characteristics of the two - different art style.

In this section, we use two kinds of algorithm to classify the art style: the traditional classification method focused on recognizing the content of the image, for example, SPM (spatial pyramid matching), ScSPM (sparse coding spatial pyramid matching). Our method is denoted as"CoCBI", because it has three types of feature, e.g. color contrast, blank-leaving, the uniformity of illumination.

For a binary image classification problem, the SVM aimsto learn a decision function as following:

$$
f(x)=\sum_{i=1}^{n} w_{i} k\left(x, x_{i}\right)+b
$$

Where $\left\{\left(\mathrm{x}_{\mathrm{i}}, \mathrm{y}_{\mathrm{i}}\right)\right\}_{\mathrm{i}=1}^{\mathrm{n}}$ are the training image set, and $\mathrm{y}_{\mathrm{i}}\{-1,1\}$ is the image labels. For a test image, suppose that its feature representation is $x$, and then if the $f(x)=1$, the test image isclassified as the 
positive one, or the negative one.In this paper, we simply use SVM to decide whether one test image belongs to the Chinese wash painting or the foreign art painting.

Table1Classifcation Rates(\%) Comparison

\begin{tabular}{|c|c|c|c|}
\hline Algorithm & 30tranings & 45traings & 60traings \\
\hline SPM & 0.806667 & 0.838634 & 0.856324 \\
\hline ScSPM & 0.921429 & 0.941818 & 0.955000 \\
\hline CoCBI & 0.9571 & 0.9600 & 0.9625 \\
\hline
\end{tabular}

From the table 1, we can conclude that, compared withthe SPM, we can achieve $13 \%$ higher classification rateaveragely, and 2\% higher for ScSPM. We believed this isdue to the fact that the features we choose are comfortablefor the art style representation.

Table. 2Comparison Rates of Different Features

\begin{tabular}{|c|c|c|c|}
\hline Algorithm & 30tranings & 45traings & 60 traings \\
\hline Colordiff & 0.6500 & 0.60000 & 0.7500 \\
\hline blank-leaving & 0.6285 & 0.6363 & 0.6500 \\
\hline Illumination & 0.9214 & 0.9090 & 0.9000 \\
\hline CoCBI & 0.9571 & 0.9600 & 0.9625 \\
\hline
\end{tabular}

Meanwhile, one single feature could just gain accuracy up to $92 \%$ at the high case and $60 \%$ at the low case. This is because CoCBI represents the image art style using 3 different features, while only single feature is not enough to describe the art style of one image.
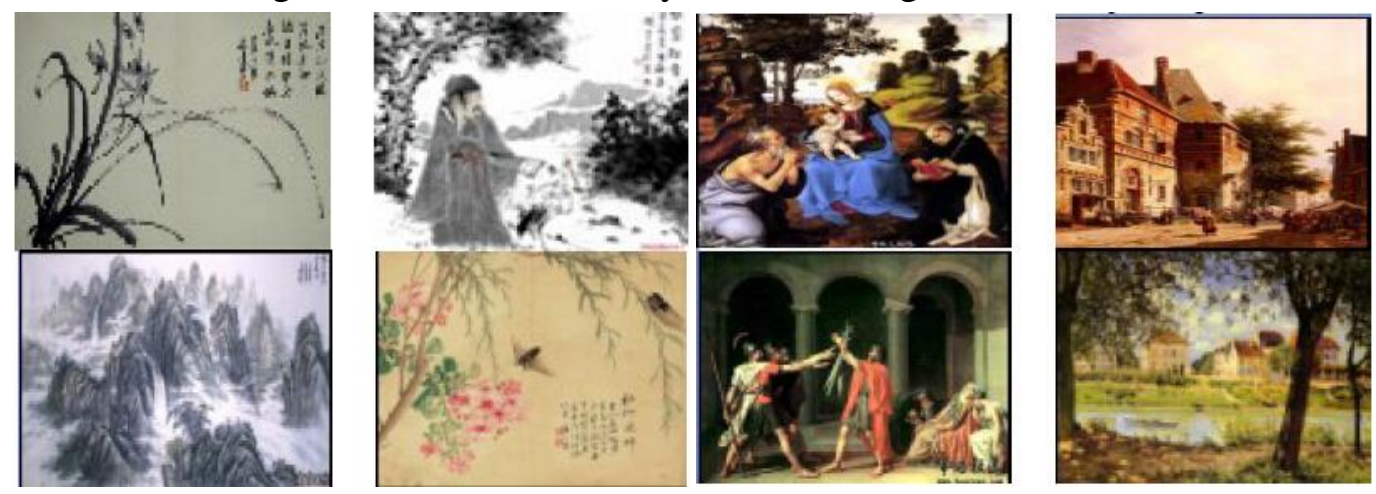

Figure.2Chinese wash paintings \& foreign art paintings

\section{Conclusions}

In this paper, we have proposed a simple but effectiveapproach to classify the art style between Chinese washpaintings and foreign art paintings. In this paper, we try toclassify art style from the view of painting techniques. Basedon careful studies on art literature, we develop three novelfeatures used in paintings to represent the art style of image,i.e. color difference, blank-leaving, uniformity ofillumination. Then, we use basic SVM to serve as theclassifier. Our experiments show that, compared with stateof-the-art approaches, not only the classification accuracyhave been improved, but also the cost times are saved a lot.Using the cues presented in this paper, our method cansuccessfully identify whether one painting belongs toChinese or foreign art style. Although the art stylereorganization is simplified into a two-class classificationtask in this paper, the presentation of art style for paintingswill be the future work. 


\section{Acknowledgement}

This work was supported by the Liaoning Provincial Education Department (W2013280); Scientific Research Foundation for the Returned Overseas Chinese Scholars, State Education Ministry; Research Fund for the Doctoral Program of Dalian University.

\section{References}

[1] M. Ding and R. F. Tong, "Content-aware copying and pasting in images," The Viusal Computer, vol. 26 , no.6-8, pp. 721-729, 2010

[2] D. M. Bradley and J. A. Bagnell, "Differential sparse coding," in NIPS, 2008.

[3] G. Edelman, Second Nature: Brain Science and Human Knowledge,Yale University Press, 2006.

[4] D.G.Stork, "Computer analysis of lighting style in fine art:Steps towards inter-artist studies,". 2011.

[5] W. D. Meng, "The artistic style and influence of Wangwei's ink paintings," Traditional Chinese Painter, pp. -72-73, 2011.

[6] M. Yelizaveta, C.Tat-Seng, and R. Jain, "Semi-supervised annotation of brushwork in paintings domain using serial combinations of multiple experts," ACM Multimedia , 2006:

[7] L. Leslie, T. S.Chua, and R. Jain, "Annotation of paintings with high level semantic concepts using transductive inference and ontology based concept disambiguation," ACM Multimedia, 2007.

[8] H. F. Li, J. He and L.P. Jiao, "Image emotional semantic classification based on color features," Computer Applications. vol. 27, no. 2, pp.453-455, 2008

[9] K. Bai, "The blank-leaving and its origin of Chinese painting," Journal of Shandong Academy of Arts, no.1, pp. 19-21, 2008

[10] S. Lazebnik, C. Schmid ,and J. Ponce, "Beyond bags of features: Spatial pyramid matching for recognizing natural scene categories," Proc.of CVPR'06, 2006 